\title{
Nodal Domains Statistics: A Criterion for Quantum Chaos
}

\author{
Galya Blum, Sven Gnutzmann, and Uzy Smilansky \\ The Weizmann Institute of Science, 76100 Rehovot, Israel
}

(Received 25 September 2001; published 1 March 2002)

\begin{abstract}
We consider the distribution of the (properly normalized) numbers of nodal domains of wave functions in $2 \mathrm{D}$ quantum billiards. We show that these distributions distinguish clearly between systems with integrable (separable) or chaotic underlying classical dynamics, and for each case the limiting distribution is universal (system independent). Thus, a new criterion for quantum chaos is provided by the statistics of the wave functions, which complements the well-established criterion based on spectral statistics.
\end{abstract}

DOI: $10.1103 /$ PhysRevLett.88.114101

The attempts to discern the "fingerprints" of the underlying classical dynamics in the patterns observed in the quantum wave functions is one of the main themes of quantum chaos [1-6]. Here, we present a new approach which is based on the distribution of the number of the nodal domains of wave functions. We show that this distribution makes a clear distinction between integrable (separable) and chaotic systems, and we study the universal features associated with each of the cases. As a convenient paradigm we consider "quantum billiards" in 2D whose classical dynamics correspond to geodesic flows with specular reflections on the domain boundaries. The corresponding Schrödinger (Helmholtz) equation reads

$$
-\triangle \Psi(\mathbf{r})=E \Psi(\mathbf{r}) ; \quad \mathbf{r} \in \Omega,
$$

where $\Omega$ is a connected compact domain on a $2 \mathrm{D}$ Riemannian manifold, and $\triangle$ is the corresponding Laplacian. If $\Omega$ has boundaries, Dirichlet boundary conditions are assumed throughout.

Considering only real solutions of (1), the nodal lines are the zero sets of $\Psi(\mathbf{r})$. The nodal domains are the connected domains where $\Psi(\mathbf{r})$ has a constant sign. The distribution of the number of nodal domains is the subject of the present note. Courant [7] was the first to address this counting problem. Ordering the eigenfunctions by the magnitude of the corresponding eigenvalues, and denoting by $\nu_{n}$ the number of nodal domains of the $n$th eigenfunction, Courant proved that for $\Omega \in \mathbb{R}^{2}$

$$
\nu_{n} \leq n \text {. }
$$

Pleijel [8] showed that the equality in (2) holds only for a finite sequence of eigenfunctions, and that

$$
\varlimsup_{n \rightarrow \infty} \frac{\nu_{n}}{n} \leq\left(\frac{2}{j_{1}}\right)^{2} \approx 0.691,
$$

where $j_{1}$ is the first zero of the Bessel function $J_{0}(x)$.

Here, we associate to each wave function the normalized nodal-domain number

$$
\xi_{n}=\frac{\nu_{n}}{n}, \quad 0<\xi_{n} \leq 1,
$$

and study their distribution, defined in the following way. Consider the spectral interval $I_{g}(E)=[E, E+g E],(g>$
PACS numbers: 05.45.Mt, 03.65.Ge

0 arbitrary), and denote by $N_{I}$ the number of eigenvalues in $I_{g}(E)$. The $\xi$ distribution associated with $I_{g}(E)$ is

$$
P\left[\xi, I_{g}(E)\right]=\frac{1}{N_{I}} \sum_{E_{n} \in I_{g}(E)} \delta\left(\xi-\frac{\nu_{n}}{n}\right) .
$$

We shall show the existence of the limiting distribution,

$$
P(\xi)=\lim _{E \rightarrow \infty} P\left[\xi, I_{g}(E)\right],
$$

and present its universal features.

For domains with boundaries, we also study the distribution of the number of nodal intersections with the boundary, denoted by $\tilde{\nu}_{n}$. This is the number of times the normal derivative at the boundary vanishes. In this case, the appropriate normalized parameter is

$$
\eta_{n}=\frac{\tilde{\nu}_{n}}{\sqrt{n}}
$$

and we define the distribution $\tilde{P}\left(\eta, I_{g}(E)\right)$ and the limiting distribution $\tilde{P}(\eta)$ as in (5) and (6), respectively.

We shall first discuss integrable systems, and in particular, those generic integrable systems which are also separable [2]. This set of systems includes the rectangular and elliptic domains in $\mathbb{R}^{2}$, surfaces of revolution [9], and Liouville surfaces [10]. In the two latter cases, we shall deal only with surfaces for which exist global action-angle variables for the geodesic flows. The relevant common features to the systems mentioned above are that (a) the Hamiltonian $H\left(I_{1}, I_{2}\right)$ is a homogeneous function of degree 2 of the action variables $I_{1,2}$ (which are expressed in units of $\hbar$ ); (b) the contour line $\Gamma$ defined by $H\left(I_{1}, I_{2}\right)=1$ in the positive quadrant of the $\left(I_{1}, I_{2}\right)$ plane is monotonic. In the semiclassical limit of interest here, the spectrum is given by the EBK quantization: $E_{l, m}=H\left(l+\alpha_{1}, m+\alpha_{2}\right)+\mathcal{O}(\sqrt{E})$ where $l, m$ are the integer quantum numbers and $\alpha_{1,2}$ are the Maslov indices. The normalized number of nodal domains (4) is $\xi_{l, m}=$ $\nu_{l, m} / n_{l, m}$. Separability implies $\nu_{l, m}=l m+\mathcal{O}(1)$, and $n_{l, m}=\mathcal{N}\left(E_{l, m}\right)$, where $\mathcal{N}(E)$ is the spectral counting function. To leading order $\mathcal{N}(E)$ can be replaced by the first term in the Weyl series, $\mathcal{N}(E)=\mathcal{A} E\left(1+\mathcal{O}\left(\frac{1}{\sqrt{E}}\right)\right)$, where $\mathcal{A}$ is the area enclosed by $\Gamma$ and the $I_{1}, I_{2}$ axes. Substituting these approximate values in (5), and converting 
the discrete sum into an integral, incurs a relative error of order $\mathcal{O}\left(\frac{1}{\sqrt{E}}\right)$. Thus,

$$
\begin{aligned}
P\left(\xi ; I_{g}(E)\right)= & \frac{1}{g E \mathcal{A}} \int_{H\left(I_{1}, I_{2}\right) \in I_{g}} d I_{1} d I_{2} \\
& \times \delta\left(\xi-\frac{I_{1} I_{2}}{\mathcal{A} H\left(I_{1}, I_{2}\right)}\right)+\mathcal{O}\left(\frac{1}{\sqrt{E}}\right) .
\end{aligned}
$$

The homogeneity of the Hamiltonian function leads to an expression for $P\left(\xi, I_{g}(E)\right)$ which is independent of both $E$ and $g$. This establishes the existence of the limiting distribution (6). Changing the integration variables $\left(I_{1}, I_{2}\right) \rightarrow(\mathcal{E}, s)$, where $\mathcal{E}=H\left(I_{1}, I_{2}\right)$ and $s$ is the arc length along the line $\Gamma,(8)$ is reduced to

$$
P(\xi)=\frac{1}{\mathcal{A}} \int_{\Gamma} d s \delta\left(\xi-\frac{I_{1}(s) I_{2}(s)}{\mathcal{A}}\right) .
$$

This form allows a simple geometrical interpretation: $I_{1}(s) I_{2}(s)$ is the area of the rectangle generated by a point $\left(I_{1}, I_{2}\right)$ on $\Gamma$ and its projections on the axes. Thus, $P(\xi)$ is the probability distribution of the areas of rectangles scaled by $\mathcal{A}$, the area between $\Gamma$ and the axes. However, $\Gamma$ is monotonic, and therefore the scaled areas are all smaller than 1 . Thus, $P(\xi)=0$ for $\xi \geq \xi_{m}$, where $\xi_{m}$ is the maximum value of the scaled areas. The monotonicity of $\Gamma$ ensures the existence of an explicit function for $\Gamma: I_{2}=I_{2}\left(I_{1}\right)$, in terms of which the integral (9) can be computed for every value of $\xi \leq \xi_{m}$. The resulting distribution is

$$
P(\xi)= \begin{cases}0, & \xi \geq \xi_{m}, \\ \left.\frac{1}{2} \sum \frac{I_{2}\left(I_{1}\right)-I_{2}^{\prime}\left(I_{1}\right) I_{1}}{I_{2}\left(I_{1}\right)+I_{2}^{(}\left(I_{1}\right) I_{1}}\right|_{\xi=\left[I_{1} I_{2}\left(I_{1}\right)\right] / \mathcal{A}}, & \xi<\xi_{m} .\end{cases}
$$

The sum is over all the real values of $I_{1}$ which satisfy $\xi=\frac{I_{1} I_{2}\left(I_{1}\right)}{\mathcal{A}}$. In the vicinity of $\xi_{m}$, typically two solutions coalesce, leading to a square root singularity of $P(\xi)$ near $\xi_{m}$. The vanishing of $P(\xi)$ in the interval $\left(\xi_{m}, 1\right)$, and the square root singularity at $\xi_{m}$ are the universal features which characterize the nodal domain distributions for separable, integrable systems.

The distribution $\tilde{P}(\eta)$ can be derived in a similar way, the only difference being that $\tilde{\nu}_{n}$ is twice the value of one of the action variables $I_{1}$, say. (The rectangular billiard is the only exception, where $\tilde{\nu}_{n}=2\left(I_{1}+I_{2}\right)$, for $I_{1,2}>$ $1)$. The scaling with respect to $\sqrt{n}$ is naturally called for. Denoting by $I_{1}^{(m)}$ the intersection of $\Gamma$ with the $I_{1}$ axis, the maximum value of $\eta$ is $\eta_{m}=2 I_{1}^{(m)} / \sqrt{\mathcal{A}}$. Then, the analog of (10) reads

$\tilde{P}(\eta)= \begin{cases}0, & \eta \geq \eta_{m}, \\ \left.\frac{1}{4 \sqrt{\mathcal{A}}}\left[I_{2}\left(I_{1}\right)-I_{2}^{\prime}\left(I_{1}\right) I_{1}\right]\right|_{I_{1}=\eta \sqrt{\mathcal{A}} / 2}, & \eta<\eta_{m} .\end{cases}$

Near $\eta_{m}, \tilde{P}(\eta)$ reflects the way that $\Gamma$ intersects the $I_{1}$ axis, and it is not universal.

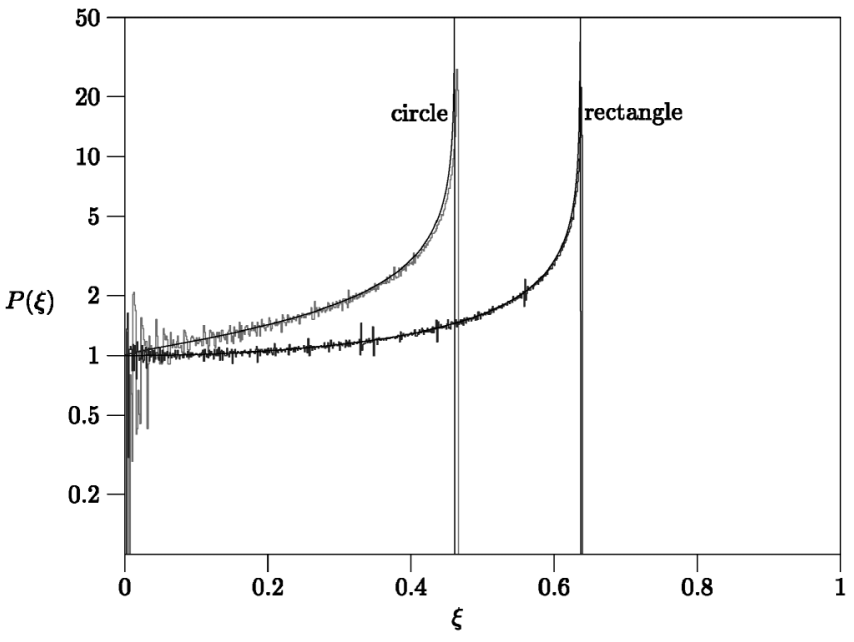

FIG. 1. The nodal domains distributions (9) for the rectangular and circular billiards in the spectral intervals: Rectangle: $62500 \leq n \leq 125000$; circle: $30000 \leq n \leq 60000$. Smooth line: the limiting distributions (10).

Numerical simulations for the rectangular and the circular domains are shown in Figs. 1 and 2, together with the predicted limiting distributions. The deviations can be explained by considering the next to leading contributions in $1 / \sqrt{E}$. The singularities seen in the numerical data mark the contributions of periodic orbits [11].

The most important feature of wave functions corresponding to separable systems is that the nodal set forms a simple grid of intersecting nodal lines. In chaotic systems, nodal intersections may occur, and when $q$ nodal lines intersect at a point, they do so at angles $\frac{\pi}{q}$. The intersections are rare, and are unstable against small perturbations. There is no known analytical expression for the number of nodal domains, and therefore we cannot offer an expression for the limiting distribution of the normalized numbers of nodal domains for chaotic billiards. However, we shall present physical arguments augmented by numerical evidence which support the conjecture that the nodal domains of random waves reproduce the observed distributions of

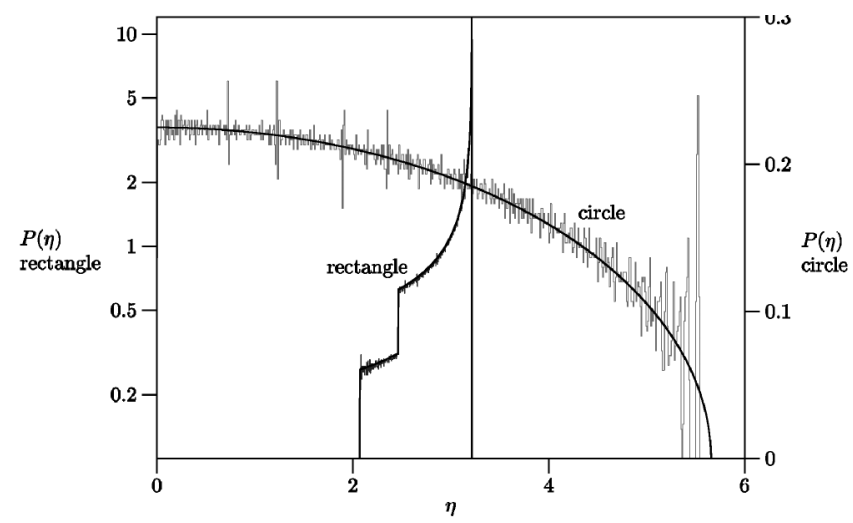

FIG. 2. The distribution of the normalized numbers of boundary intersections for the rectangular and circular billiards, in the same spectral intervals as in Fig. 1. Smooth line: the limiting distributions (11). 
chaotic billiards [3]. While this work was in progress, Bogomolny and Schmit [12] proposed a method to compute the nodal domain distributions for random waves, based on a percolation model. We shall compare our numerical result to their predictions.

The scattering approach to the quantization of billiards $[13,14]$ implies that the wave function can be well approximated by

$$
\Psi_{n}(\mathbf{r}) \approx \sum_{l=-L}^{L} a_{l} J_{l}\left(k_{n} r\right) e^{i l \theta}, \quad a_{-l}=a_{l}^{*}(-1)^{l} .
$$

Here, $k=\sqrt{E}, L=[k \mathcal{L} / 2 \pi]$, and $\mathcal{L}$ is the boundary length. The $2 L+1$ coefficients $\left\{a_{l}\right\}$ are the eigenvector of the scattering matrix $S\left(k=k_{n}\right)$ corresponding to an eigenvalue 1 . The $S$ matrix is the semiclassical quantization of the billiard bounce map. For hyperbolic billiard maps, quantum ergodicity implies that the vectors $\left\{a_{l}\right\}$ are uniformly distributed on the sphere. For large $L$, this distribution can be approximated by considering $\left\{\operatorname{Re}\left(a_{l}\right), \operatorname{Im}\left(a_{l}\right)\right\}$ as independent random Gaussian variables with $\left\langle\left|a_{l}\right|^{2}\right\rangle=1$. Hence, the set of eigenfunctions (12) is conjectured to be simulated by the Gaussian random wave ensemble.

To check the conjectured relation between wave functions of chaotic billiards and the ensemble of random waves, we computed the lowest 1637 (1483) eigenfunctions of the stadium (Sinai) billiards. The nodal domains of the billiard wave functions were counted using the Hoshen-Kopelman method [15]. We performed the following tests:

(i) The distribution of boundary intersections. - Given a function of the type (12) we computed the number of the zeros of its normal derivative on a circle of radius $R(L=k R)$. Denoting the normal derivative by $u(\theta)=$ $\left.\partial_{r} \Psi(r, \theta)\right|_{r=R}$ the number of its zeros is [16]

$$
\tilde{\nu}_{u}=\int_{0}^{2 \pi} d \theta \int_{-\infty}^{\infty} \int_{-\infty}^{\infty} \frac{d \xi d \eta}{2(\pi \eta)^{2}} e^{i \xi u(\theta)}\left(1-e^{i \eta \dot{u}(\theta)}\right),
$$

where $\dot{u}(\theta)=d_{\theta} u(\theta)$. The mean number of zeros $\langle\tilde{\nu}\rangle$, and its variance were evaluated by performing the Gaussian integrals, with the result

$$
\begin{aligned}
\langle\tilde{\nu}\rangle & =k R \sqrt{1+\left(\frac{2}{k R}\right)^{2}} \approx k R \approx \frac{k \mathcal{L}}{2 \pi}, \\
\operatorname{var}(\tilde{\nu}) & \approx 0.0769 k \mathcal{L} .
\end{aligned}
$$

The expression for $\langle\tilde{\nu}\rangle$ is exact, while that for the variance is the leading term in the semiclassical limit. The variance was computed analytically up to an integral whose numerical value is quoted. Since for a billiard of area $A$, $n=\frac{A k^{2}}{4 \pi}$, (14) supports the proposed scaling (7). Moreover, it implies that the distribution of the scaled number of nodal intersections limits to a delta function centered at $\eta \frac{\sqrt{\pi A}}{\mathcal{L}}=1$. The agreement between the billiards data

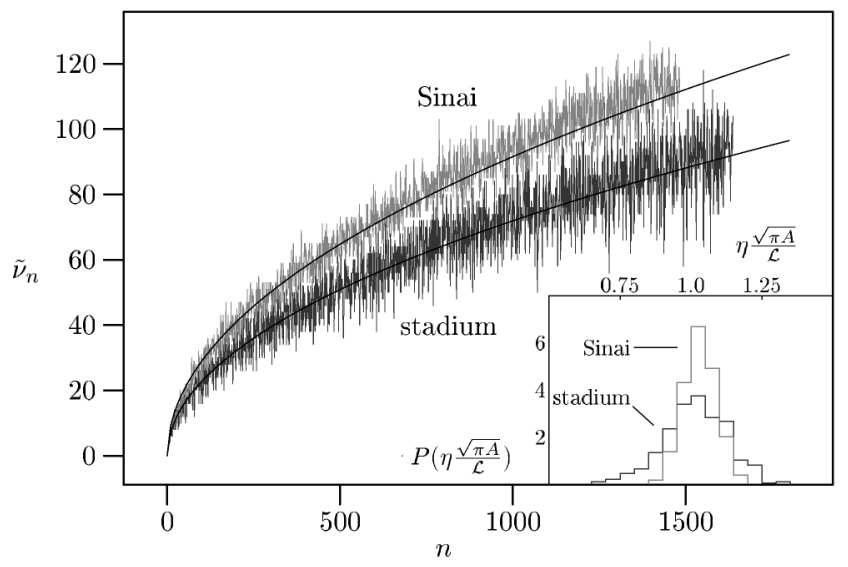

FIG. 3. The number of boundary intersections $\tilde{\nu}_{n}$ as a function of $n$ for the stadium and the Sinai billiards. Smooth line: the random waves result (14). Inset: the $\eta \frac{\sqrt{\pi A}}{\mathcal{L}}$ distribution.

shown in Fig. 3 and the predictions of the random waves model, lends very strong support to our conjecture. However, the fluctuations in $\nu_{n}$ are affected by the presence of "bouncing ball" states, which explains the apparent difference between the widths of the $\eta$ distributions for the two billiards.

(ii) Signed area distribution. - Denoted by $|\Omega(\Psi)|_{ \pm}$, the total area where the wave function is positive (negative). Clearly $\left\langle|\Omega|_{ \pm}\right\rangle=|\Omega| / 2$, where $|\Omega|$ is the billiard area. To compute the signed area variance $\left\langle\left(|\Omega|_{+}-\right.\right.$ $\left.\left.|\Omega|_{-}\right)^{2}\right\rangle /|\Omega|^{2}$ we used the identity

$$
|\Omega(\Psi)|_{ \pm}=\frac{1}{2 \pi i} \lim _{\epsilon \rightarrow 0^{+}} \int_{\Omega} d^{2} r \int_{-\infty}^{\infty} d \xi \frac{e^{ \pm i \xi \Psi(\mathbf{r})}}{\xi-i \epsilon} .
$$

For a circle of radius $R$ the signed area variance can be reduced to an expression involving a single integral which was evaluated numerically, and is shown in Fig. 4 as a function of $n=(R k / 2)^{2}$. For large $n$,

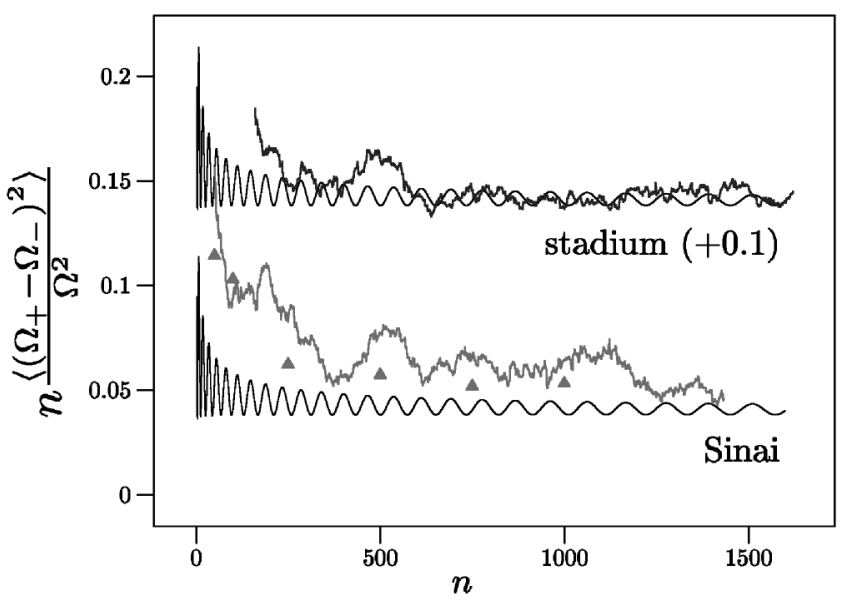

FIG. 4. The normalized signed area variance for the stadium and Sinai billiards (running averages). Smooth curve: analytic expression for random waves. Triangles: numerical simulation for random waves (see text). 


$$
\frac{1}{|\Omega|^{2}}\left\langle\left(|\Omega|_{+}-|\Omega|_{-}\right)^{2}\right\rangle \approx 0.0386 n^{-1} .
$$

Figure 4 shows also the data for the stadium and the Sinai billiards, which converge to the asymptotic limit (16). The slow convergence of the Sinai data is due to the exsitence of corners with sharp angles in the desymmetrized Sinai billiard. The effect of such corners on the wave function is accentuated at low energies, and we checked this effect, by computing the variance in a random wave model for which the only angular waves allowed are multiples of four (triangular dots). This simulates the boundary conditions on the symmetry line of the Sinai billiard, and shows similar slow convergence to the asymptotic value.

(iii) The number of nodal domains $\nu_{n}$. - The number of nodal domains for the stadium and Sinai billiards is plotted in Fig. 5. The predicted $\left\langle\nu_{n}\right\rangle$ for the random waves model are shown as large circular dots. These data were computed numerically by sampling the random wave ensemble, assuming a square domain. The agreement between the billiards and the random waves results is satisfactory, and confirms the expected scaling $\nu_{n} \approx n$ for high $n$. The random and the billiards data sets, however, show clear deviations from the expected asymptotic scaling for low $n$ values, where $\nu_{n} \approx n^{1 / 2}$. To check the reason for this behavior we computed and plotted the number of interior nodal domains - those domains whose boundaries do not include the billiard boundaries. Their number for the billiards and the random waves model scale as $n$, suggesting the following explanation: At low $n$, most of the domains are boundary domains whose number scales as the number of boundary intersections, that is, $n^{1 / 2}$. Only when the wavelengths are sufficiently smaller than the typical linear dimension of the billiard, the majority of domains are interior, and their number increases linearly with $n$. The smooth lines in Fig. 5 represent the analytical result

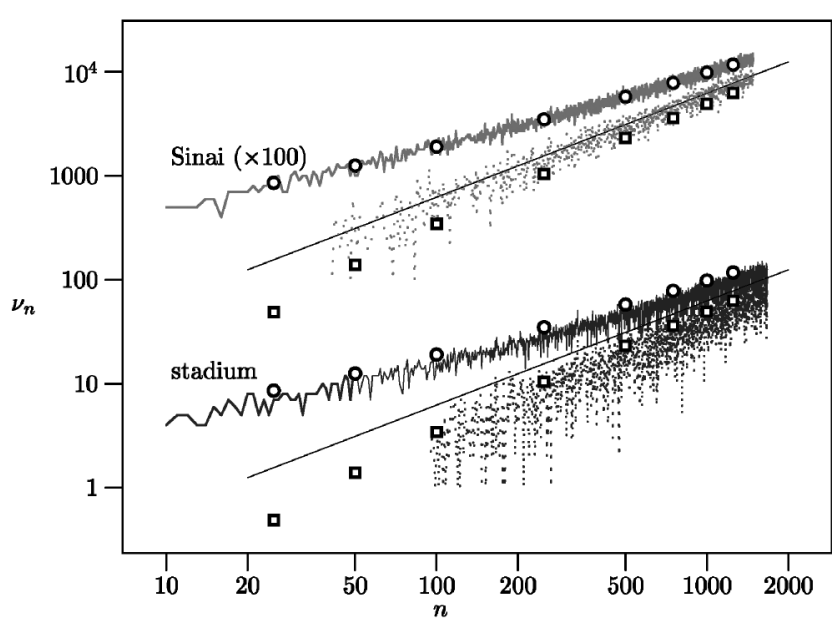

FIG. 5. The number of nodal domains $\nu_{n}$ (solid lines) and inner nodal domains (dotted) for the Sinai and the stadium billiards, and for random waves (circles for nodal domains and squares for interior nodal domains). Smooth lines: the prediction of the theory of Bogomolny and Schmit. of [12] for $\left\langle\nu_{n}\right\rangle$. This theory does not include the effects of the boundaries, and therefore it applies in the limit of high $n$. It is consistent with our numerical data, and confirms the linear scaling with $n$. The numerical simulations based on the random waves model, supported by the results of [12] predict that $\operatorname{var}\left(\nu_{n}\right)$ scales linearly with $n$ for large $n$. Thus, the limiting distribution $P(\xi)$ for chaotic billiards is expected to limit to a delta function, centered at the value predicted by [16] to be 0.0624 , much less than the Pleijel bound, or the values observed for the square and circle billiards.

To summarize, we provided here ample evidence to show that the study of nodal domains counts provides a clear criterion of quantum chaos, which is not directly related to spectral statistics. Our results add a new dimension to previous attempts to attribute the morphology of wave functions to the nature of the underlying classical dynamics $[1-6]$.

This work was supported by the Minerva Center for nonlinear Physics at the Weizmann Institute. S. G. acknowledges support from the Minerva Foundation. We thank E. Bogomolny and C. Schmit for allowing the use of their results prior to publication, and to Y. Avron, Z. Rudnick, Y. Sinai, M. Solomiak, and D. Volk for stimulating discussions and comments.

[1] A. I. Shnirelman, Usp. Mat. Nauk 29, 181 (1974).

[2] M.C. Gutzwiller, Chaos in Classical and Quantum Mechanics (Springer-Verlag, New York, 1990), Chap. 15.

[3] M. V. Berry, J. Phys. A 10, 2083-2091 (1977).

[4] E. Heller, in Chaos and Quantum Physics, Proceedings of the Les Houches Summer School, Session LII, edited by M.-J. Giannoni, A. Voros, and J. Zinn-Justin (Elsevier Science, New York, 1991).

[5] R. Aurich et al., Physica (Amsterdam) 64D, 185 (1993); 129D, 169 (1999).

[6] F. Simmel and M. Eckert, Physica (Amsterdam) 97D, 517 (1996).

[7] R. Courant, Nachr. Ges. Wiss. Göttingen, Math. Phys. Kl. (1923).

[8] A. Pleijel, Commun. Pure Appl. Math. 9, 543 (1956).

[9] P. M. Bleher, Duke Math. J. 74, 45-73 (1994).

[10] D. V. Kosygin, A. A. Minasov, and Ya. G. Sinai, Russ. Math. Surv. 48, 1-142 (1993).

[11] G. Blum, S. Gnutzmann, and U. Smilansky (to be published).

[12] E. Bogomolny and C. Schmit, Phys. Rev. Lett. 88, 114102 (2002).

[13] U. Smilansky, in Mesoscopic Quantum Physics, Proceedings of the Les Houches Summer School, Session LXI, edited by E. Akkermans, G. Montambaux, and J. L. Pichard (Elsevier Science, New York, 2002).

[14] D. Klakow and U. Smilansky, J. Phys. A 29, 3213 (1996).

[15] J. Hoshen and R. Kopelman, Phys. Rev. B 14, 3438-3445 (1976).

[16] M. Kac, Am. J. Math. 65, 609-615 (1943). 